Eliciting von Neumann-Morgenstern Utilities When Probabilities are Distorted or Unknown Author(s): Peter Wakker and Daniel Deneffe

Source: Management Science, Vol. 42, No. 8 (Aug., 1996), pp. 1131-1150

Published by: INFORMS

Stable URL: http://www.jstor.org/stable/2634448

Accessed: $27 / 04 / 2011$ 04:35

Your use of the JSTOR archive indicates your acceptance of JSTOR's Terms and Conditions of Use, available at http://www.jstor.org/page/info/about/policies/terms.jsp. JSTOR's Terms and Conditions of Use provides, in part, that unless you have obtained prior permission, you may not download an entire issue of a journal or multiple copies of articles, and you may use content in the JSTOR archive only for your personal, non-commercial use.

Please contact the publisher regarding any further use of this work. Publisher contact information may be obtained at http://www.jstor.org/action/showPublisher?publisherCode=informs.

Each copy of any part of a JSTOR transmission must contain the same copyright notice that appears on the screen or printed page of such transmission.

JSTOR is a not-for-profit service that helps scholars, researchers, and students discover, use, and build upon a wide range of content in a trusted digital archive. We use information technology and tools to increase productivity and facilitate new forms of scholarship. For more information about JSTOR, please contact support@jstor.org. 


\title{
Eliciting von Neumann-Morgenstern Utilities When Probabilities Are Distorted or Unknown
}

\author{
Peter Wakker - Daniel Deneffe \\ Medical Decision Making Unit, University of Leiden, Leiden, The Netherlands \\ The Fuqua School of Business, Duke University, Durham, North Carolina 27706 \\ and Management Education Institute, Arthur D. Little. Inc., Brussels, Belgium
}

$\mathrm{T}$ his paper proposes a new method, the (gamble-)tradeoff method, for eliciting utilities in decision under risk or uncertainty. The elicitation of utilities, to be used in the expected utility criterion, turns out to be possible even if probabilities are ambiguous or unknown. A disadvantage of the tradeoff method is that a few more questions usually must be asked to clients. Also, the lotteries that are needed are somewhat more complex than in the certaintyequivalent method or in the probability-equivalent method. The major advantage of the tradeoff method is its robustness against probability distortions and misconceptions, which constitute a major cause of violations of expected utility and generate inconsistencies in utility elicitation. Thus the tradeoff method retains full validity under prospect theory, rank-dependent utility, and the combination of the two, i.e., cumulative prospect theory.

The tradeoff method is tested for monetary outcomes and for outcomes describing lifeduration. We find higher risk aversion for life duration, but the tradeoff method elicits similar curvature of utility. Apparently the higher risk aversion for life duration is due to more pronounced deviations from expected utility.

(Utility Measurement; Probability Distortion; Prospect Theory; Decision Analysis; Risk Aversion; Standard Gamble)

\section{Introduction}

One of the most disturbing factors in the application of expected utility is that the existing methods of utility elicitation yield systematically different results (Hershey and Schoemaker 1985, Nord 1992). The literature recognizes a number of disadvantages of each of these methods. Of the three most common methods, the certainty-equivalent method and the probabilityequivalent method require precise knowledge of probabilities. They thus suffer from an important cause of the violation of expected utility, namely the misconception of probabilities. The third method, direct scaling, does not invoke probabilities. Its validity is, however, questionable. There is no reason to believe that the obtained scale values are the utilities to be used in an expectation criterion. A priori, they could be any (nonlinear) transform thereof. A fourth method, less often used in applications, is the lottery-equivalent method. It shares several characteristics with the method introduced in this paper, but still requires precise knowledge of probabilities.

Several variations of the above methods have been described (an early survey was given in Farquhar 1984) but no new, independent method has been proposed in 
many years. This paper precisely suggests a new method of utility elicitation, the "gamble-tradeoff method," or "tradeoff method" for short. This method uses, in the context of uncertainty, a technique that has been used previously in multiattribute utility theory (the "saw-tooth" method in Fishburn 1967; Johnson 1974; Keeney and Raiffa 1976, §3.4.7; Kirkwood and Sarin 1980; von Winterfeldt and Edwards 1986), conjoint measurement theory (Krantz et al. 1971; Tversky et al. 1988), and in axiomatizations of several risktheory forms (Wakker 1984, 1989, 1994; Tversky and Kahneman 1992; Wakker and Tversky 1993). The main advantage of the tradeoff method is that it minimizes the role of probabilities while preserving full validity when used in the expected utility criterion. Utilities can be generated when a subject distorts or misperceives probabilities, or when the subject does not understand probabilities. In the latter case, general events whose probabilities need not be known with precision can be used to elicit utilities.

We would like to emphasize that we are in no way arguing that eliciting utilities with unknown probabilities is in general superior to eliciting utilities with known probabilities. Rather, we claim only that the tradeoff method enables one to elicit utilities without invoking known probabilities and that this can offer a number of advantages. The tradeoff method can perfectly well be applied when probabilities are known. The method then still provides more robustness against deviations from expected utility than existing methods.

Both under the rank-dependent deviations from expected utility (Quiggin 1981; Schmeidler 1989) and under prospect theory (Kahneman and Tversky 1979), does the tradeoff method not only provide robustness against probability distortions, but it even completely eliminates the effects of probability distortions. Thus, under these theories, the method generates the correct utilities. In particular the method can be applied to gains and losses in "cumulative prospect theory" (Tversky and Kahneman 1992), which is the modern version of prospect theory. The desirability of developing elicitation methods that remain valid for nonexpected utility models has been pointed out several times (Farquhar 1984; Loomes and McKenzie 1989; Hogarth and Einhorn
1990, p. 799; Birnbaum and Sutton 1992; Weber 1994). Tversky and Kahneman $(1992, \S 2.3)$ described the estimation of a complex choice model, such as cumulative prospect theory, as a problematic issue.

Another feature of our method is that it can uncover deviations from expected utility through inconsistencies in the revealed tradeoff comparisons. Thus, the method is suited for axiomatizations of expected utility models and the rank-dependent generalizations thereof; see Wakker and Tversky (1993), Wakker (1994), and the references therein. Finally, the elicitation method can be used to elicit probabilities indirectly. The entire expected utility model can then be elicited without prior knowledge of any probability or utility.

We developed the tradeoff method primarily to be applied in prescriptive decision analysis. It is then used to construct a rational and truly representative utility, where inconsistencies are to be resolved rather than accepted. The method is suited for prescriptive applications because it allows for convenient cross-checkings, and because it appeals directly to a rational decision approach: the weighing of arguments.

The paper is organized as follows. Section 2 contrasts the tradeoff method with existing methods of utility elicitation. Section 3 describes two experiments in which utilities are elicited by means of our method, one for monetary outcomes, the other for outcomes that describe life-duration. It has often been found in the literature that risk attitudes depend on the domain and the context. We find higher risk aversion for life durations than for money. By the tradeoff method, curvature of utility can be disentangled from other aspects of risk attitude. Thus we find that the higher risk aversion for life duration is not due to curvature of utility but to more pronounced deviations from expected utility. Section 4 shows that the tradeoff method can elicit utility for a number of nonexpected utility theories. Sections 5 and 6 discuss advantages and disadvantages of the method. Conclusions appear in \$7.

\section{Existing Methods of Utility Elicitation, and the New Method}

The most fruitful model for normative decision under uncertainty is the expected utility (EU) model. Its first 
axiomatic foundation has been laid down by Savage (1954). Normative objections were raised by Allais (1953), Machina (1982), and several others. The current interest in nonexpected utility models stems from the descriptive inadequacy of EU. Several non-EU models have been developed. Surveys are given by Machina (1987), Fishburn (1988), Karni and Schmeidler (1990), and Kischka and Puppe (1992). None of these models has as yet reached the stage of being operational for normative applications, let alone of being viewed as superior to EU. As a result, the dominant view still seems to be that EU is the proper normative model. This model is also the point of departure of our paper. It should, however, be recognized that eliciting utilities from clients is a descriptive activity, so that prescriptive applications still have to reckon with deviations from expected utility. This consideration motivated the development of a robust method.

In EU, probabilities measure uncertainties, and von Neumann-Morgenstern (vNM) utilities are used to evaluate outcomes. Decisions are subsequently made according to expectations of utility. The probabilities are often obtained from experts and utilities are elicited from clients.

We concentrate on holistic utilities and do not discuss the decomposition of utility into separate dimensions of outcomes (price, fuel consumption, maximum speed, etc.); see Keeney and Raiffa (1976) and Dyer et al. (1992). For simplicity of the exposition, we assume that outcomes are real numbers and that monotonicity holds (more is preferred to less). We first discuss existing methods of utility elicitation and then describe the new method.

The three most frequently used methods are direct scaling, the certainty-equivalent method, and the probability-equivalent method. A fourth method, the lottery-equivalent method, has not been applied as often as the other methods, even though it possesses a number of advantages. Under direct scaling, the client is asked to evaluate outcomes directly on a numerical scale (or some categories), and, for instance, to assign a value of 0 to the worst outcome, a value of 100 to the best outcome, a value of 50 to an outcome that lies halfway between the best and the worst outcome, etc. Direct scaling is easy to use and thus has many practical ad- vantages. A fundamental problem, however, is that the use of direct scaling to elicit vNM utilities lacks a theoretical justification. Even if a person follows the dictum of expected utility, there is no reason to expect that the values obtained from direct scaling can be used as vNM utilities. A priori, they might be any increasing transform thereof. In addition, direct scaling has sometimes been found to be problematic in applications (Torrance 1976).

The certainty-equivalent method, the probabilityequivalent method, and the lottery-equivalent method have a sounder theoretical foundation. Specifically, if a person were truly to maximize EU and if no measurement errors were to occur, these methods would elicit vNM utilities exactly. We now discuss the certainty- and probability-equivalent methods in more detail and describe the resulting procedures for utility elicitation. These methods are used in the experiments described in $\$ 3$. The lottery-equivalent method, introduced by McCord and DeNeufville (1986), is discussed in $\S 4$. We denote by $(x, p ; z)$ the two-outcome lottery that assigns probability $p$ to outcome $x$ and probability $1-p$ to outcome $z$. In the description of the utility elicitation methods below, we first show how one equality of utilities can be derived from observed indifferences. Next we describe what we call the "basic procedure." This shows how the elicitation of some desired number of equalities of utilities can be implemented.

In the certainty-equivalent (CE) method, the analyst asks the client to compare a lottery $(x, p ; z)$ with a certain outcome. The analyst then varies the certain outcome until the client reveals indifference between the certain outcome, denoted $\mathrm{CE}(p)$, and the lottery $(x, p ; z)$. Substituting EU with the vNM utility $u$, the equality

$$
u(\mathrm{CE}(p))=p u(x)+(1-p) u(z)
$$

is obtained. The basic CE procedure for eliciting utilities is as follows. First, two outcomes $M>m$ are fixed such that the range of outcomes between them includes all outcomes of interest. For instance, $M$ may designate 80 years in perfect health, and $m$ immediate death. We assume henceforth that $m=0$. One arbitrarily sets $u(0)=0$ and $u(M)=1$. Then for any desired probability $p$ the outcome $\operatorname{CE}(p)$ is found that is 
indifferent to $(0, p ; M)$. Utility $p$ is then assigned to this outcome.

The CE method is often used in a bisection form, that only uses probabilities $\frac{1}{2}$ (von Winterfeldt and Edwards 1986 , and $\S 3$ below). First, the outcome $\operatorname{CE}\left(\frac{1}{2}\right)$ is found as above. Then an outcome denoted $\mathrm{CE}^{\prime}\left(\frac{1}{4}\right)$ is obtained through an indifference $\mathrm{CE}^{\prime}\left(\frac{1}{4}\right) \sim\left(0, \frac{1}{2} ; \mathrm{CE}\left(\frac{1}{2}\right)\right)$. Substitution of EU shows that the utility of $C E^{\prime}\left(\frac{1}{4}\right)$ must be $\frac{1}{4}$. ${ }^{1}$ An indifference $\mathrm{CE}^{\prime}\left(\frac{3}{4}\right) \sim\left(\mathrm{CE}\left(\frac{1}{2}\right), \frac{1}{2} ; M\right)$ is used to obtain the outcome $\mathrm{CE}^{\prime}\left(\frac{3}{4}\right)$, with utility $\frac{3}{4}$. Similarly one finds $\mathrm{CE}^{\prime}(j / 8)$ for $j=1,3,5,7$. For example, $\mathrm{CE}^{\prime}\left(\frac{3}{8}\right)$ is obtained from the indifference $\mathrm{CE}^{\prime}\left(\frac{3}{8}\right) \sim\left(\mathrm{CE}^{\prime}\left(\frac{1}{4}\right), \frac{1}{2} ; \mathrm{CE}\left(\frac{1}{2}\right)\right)$; etc. After a sufficient number of questions, each desired level of specification can be obtained.

In the probability-equivalent (PE) method, the analyst also asks the client to compare a lottery $(x, p ; z)$ with a certain outcome $y(x<y<z)$. In contrast to the CE method, the outcomes $x, y, z$ are now fixed and the analyst varies the probability $p$ until the client reveals indifference between the certain outcome $y$ and the lottery $(x, p ; z)$. That indifference reveals the equality $u(y)$ $=p u(x)+(1-p) u(z)$. In the basic PE procedure, one starts with a minimal outcome, say 0 , and a maximal outcome $M>0$, and sets $u(0)=0, u(M)=1$. Then for any desired certain outcome $x$ the probability $p$ is found such that $x \sim(0, p ; M)$. Consequently, the utility of $x$ must be $p$.

We now describe the gamble-tradeoff method, or tradeoff method for short. Its abbreviation is TO method. Rather than searching for an indifference between a certain outcome and a gamble, this method draws inferences from indifferences between two-outcome gambles (as is also done in the lottery-equivalent method described in \$5). For the sake of clarity of the exposition, we first explain the method for the case in which probabilities are known to the client. The client is asked to compare lotteries $(X, p ; r)$ and $(x, p ; R)$ for $X>x$ and "reference outcomes" $R>r$. The values $p, r, x$, and $R$ are fixed, and the analyst varies $X$ until the client reveals the indifference

$$
(X, p ; r) \sim(x, p ; R)
$$

\footnotetext{
${ }^{1}$ For a perfect EU maximizer $\mathrm{CE}^{\prime}\left(\frac{1}{4}\right)$ and $\mathrm{CE}\left(\frac{1}{4}\right)$ (defined before) must coincide. In general, they may differ.
}

No conclusions are drawn from one indifference of the above form. Instead, the client is asked to compare another pair of lotteries, $(Y, p ; r)$ and $(y, p ; R)$ for $Y$ $>y$ and the same reference outcomes $R>r$. Again $p$, $r, y$, and $R$ are fixed, and now $Y$ is varied until the client reveals indifference between the lotteries:

$$
(Y, p ; r) \sim(y, p ; R) .
$$

We substitute EU with the vNM utility function $u$. From the first indifference (1), the analyst derives the equality

$$
p u(X)+(1-p) u(r)=p u(x)+(1-p) u(R),
$$

thus

$$
p(u(X)-u(x))=(1-p)(u(R)-u(r)) .
$$

The second indifference implies the equality

$$
p u(Y)+(1-p) u(r)=p u(y)+(1-p) u(R),
$$

thus

$$
p(u(Y)-u(y))=(1-p)(u(R)-u(r)) .
$$

Together these equalities imply $p(u(X)-u(x))=p(u(Y)$ $-u(y))$, i.e.,

$$
u(X)-u(x)=u(Y)-u(y)
$$

Thus, the combination of indifferences has revealed an equality of utility differences that can be used for utility elicitation.

Now we describe the basic TO procedure by means of lotteries $(x, p ; y)$. The probability $p$ is fixed throughout. First the decision analyst chooses "reference" outcomes $r<R$ and specifies a minimal outcome $x_{0}$ (e.g., $\left.x_{0}=0\right)$. The analyst then asks the client for the outcome $x_{1}$ that makes the client indifferent between lotteries $\left(x_{1}\right.$, $p ; r)$ and $\left(x_{0}, p ; R\right)$. Next, the analyst asks the client for the outcome $x_{2}$ that makes the client indifferent between $\left(x_{2}, p ; r\right)$ and $\left(x_{1}, p ; R\right)$. From these two indifferences the analyst infers that $u\left(x_{2}\right)-u\left(x_{1}\right)=u\left(x_{1}\right)-u\left(x_{0}\right)$. Setting $u\left(x_{0}\right)=0$ we get $u\left(x_{2}\right)=2 u\left(x_{1}\right)$. Inductively, any $x_{j}$ is defined such that the client is indifferent between $\left(x_{j}, p\right.$; $r)$ and $\left(x_{j-1}, p ; R\right)$, which in combination with the other indifferences implies $u\left(x_{j}\right)=j \times u\left(x_{1}\right)$. This process continues until a sufficiently wide range of outcomes is covered. We can set $u\left(x_{j}\right)=j \times \sigma$ for any arbitrary positive scale parameter $u\left(x_{1}\right)=\sigma$, e.g., $\sigma=1 / n$ with $n$ denoting 
the index of the last outcome $x_{n}$. The reference outcomes $r, R$ are chosen close enough to each other so that the revealed sequence $x_{1}, \ldots, x_{n}$ is sufficiently narrow and gives utility to the desired level of accuracy.

Note that in the above calculations the only requirement on the probability $p$ is that it be strictly positive. Its value is immaterial in the calculations, and all that matters is that the same $p$ be used across different lotteries. We use this observation to extend the TO method to the case of events with unknown probabilities instead of given numerical probabilities. As uncertain events are closer to real life experience than numerical probabilities, a procedure that uses such events can be more realistic to clients than a procedure that invokes numerical probabilities.

Let $A$ be an event with unknown probability ("surgery will succeed"), and let $(X, A ; r)$ denote the gamble giving outcome " $X$ years of survival" if event $A$ obtains, and " $r$ years of survival" otherwise. This paper uses the general term gamble when probabilities are unknown. For the case where probabilities are known, the term "lottery" is used. In the example, uncertainty is not introduced through numerical probabilities, but through the uncertainty of events to which the client can relate.

Calculations are now based on the subjective expected utility model. That is, there exists some "subjective" probability for event $A$ that is used in an expected utility criterion. Indifferences

$$
(X, A ; r) \sim(x, A ; R) \text { and }(Y, A ; r) \sim(y, A ; R) \text {, }
$$

again imply $u(X)-u(x)=u(Y)-u(y)$, using similar calculations as before. The decision analyst can infer this equality without knowing the client's subjective probability of $A$. Thus the TO method generalizes existing methods. It shares with existing methods the requirement that the client's subjective probability of $A$ be constant throughout the elicitation procedure. Utilities $u\left(x_{j}\right)$ $=j \times \sigma, j=0, \ldots, n$ are now elicited through indifferences $\left(x_{j}, A ; r\right) \sim\left(x_{j-1}, A ; R\right){ }^{2}$

\footnotetext{
${ }^{2}$ Obviously, the subjective probability of event $A$ does affect the values $x$ and $y$ in the indifferences in (2). It does not, however, affect the correctness of the equality of utility differences. Similarly, in the TO experiment, the client's subjective probability does affect the elicited values $x_{1}, \ldots, x_{n}$, but it does not affect the correctness of our inference that these values are equally spaced in utility units.
}

Utilities can also be elicited when the client is informed about verbal probabilities, rather than about numerical probabilities or uncertain events. For instance, one can tell the client that "probably outcome $X$ will result, otherwise outcome $r$ will." We denote this by $(X$, $A ; r)$ where $A$ abbreviates probably. Indifferences as in (2) above imply the equality $u(X)-u(x)=u(Y)-u(y)$. That different clients may interpret "probably" differently or that the analyst may not know which probability corresponds with "probably" for a given client is immaterial. What does matter is that the client assigns the same decision weight to "probably" in different questions. This can be enhanced by pointing out to the client that "probably" indicates the same likelihood across all gambles. We thus share with existing methods the assumption that the processing of uncertainty does not vary during the elicitation procedure.

\section{Experiments}

This section describes two experiments. They are presented here first and foremost to clarify the applicability of the TO method. The results of these experiments also support the arguments presented in the following sections. Section 4 demonstrates that the TO utility elicitations reported here are also valid under rankdependent utility and some other nonexpected utility theories.

The subjects of the experiments were all acquainted with probabilities and expectations. Most of them had heard about expected utility at some point, but not shortly before the experiments. The subjects were asked to imagine that the outcomes of their decisions in the experiment affected their own life duration in the first experiment and their own monetary reward in the second one. It was emphasized that there were no right or wrong answers but rather that the subjects' answers should reflect their preferences. The experiments took about 20 minutes per subject.

Procedure. The experiments were conducted in small groups (approximately ten subjects per group) in classrooms. All items were administered with paper-andpencil questionnaires. Because the TO answers served as input for the other questions, the TO elicitations were 
always carried out first. These were followed by the CE elicitations, and finally by the PE elicitations.

\subsection{The Life-duration Experiment}

Subjects. The object of the first experiment was to elicit the utility of life duration from 54 subjects. Fifteen of them were Ph.D. students in economics who were at the beginning of a decision theory course in Copenhagen; 15 subjects were undergraduate students in psychology at the University of Leiden who were enrolled in a cognition course; and, finally, 24 subjects were medical residents at the University of Leiden who were enrolled in a decision theory course.

Method. The subjects were asked to imagine that they displayed symptoms of one of two diseases. These symptoms did not allow the subjects to distinguish which of these two diseases they actually carried. Nonetheless, they immediately had to choose to undergo one of two possible operations. The number of years the subject would survive after the operation (followed by immediate death) depended on the combination of operation and disease, as illustrated in Figure 1. The quality of life during these years would be normal. Thus outcomes are numbers of life-years in this experiment.

Stimuli for the TO elicitations. Figure 1 illustrates the generic form of the TO questions. At an earlier stage of the experiment, the gambles had been presented in a matrix format but it was found that subjects preferred the decision-tree format of Figure 1. ${ }^{3}$ Prior to the actual experiment, the subjects were asked two choice questions to familiarize themselves with the setting. For both questions, a value of 6 was first substituted for $x_{j}$ in the figure. In addition, for the first question a life duration of 20 years was substituted for $Y$ in the figure. The subjects were then asked to indicate their preferred operation. The second question was identical to the first one with the exception that 8 (instead of 20) was substituted for $Y$.

In the actual experiment, the value $x_{j}=6$ was retained for the first question. (In the notation of the previous section, this value 6 is $x_{0}$.) The value $Y=8$ was removed,

\footnotetext{
${ }^{3}$ This was also suggested by the editor.
}

Figure 1

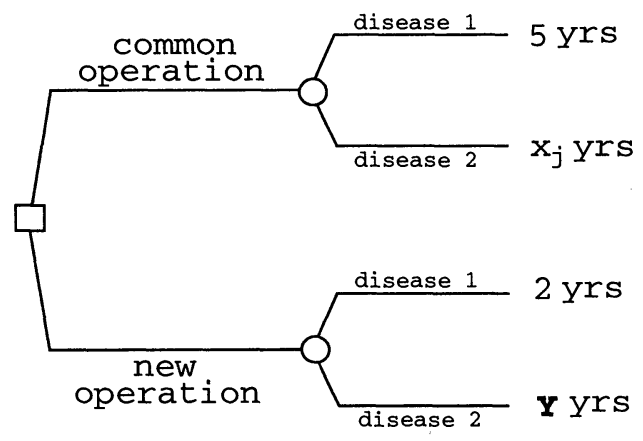

Question 1. Decide for each $0<\mathbf{Y}<100$ whether you prefer the new or the common operation.

Question 2. For large values of $\mathbf{Y}$ you probably preferred the new operation, for small values the common one. Thus, for some value of $\mathbf{Y}$, which we call $\mathbf{x}_{1}$, your preference switched from one operation to the other. Fill in this switching value on the answer sheet.

and the subjects were now asked to fill in the number of years for $Y$ that would make them indifferent between the common and the new operation; this value was called the "switching value" for their preferences. It coincides with $x_{1}$ in the notation of $\$ 2$. Next this value $x_{1}$ was substituted for $x_{j}$, and the subjects were again asked to fill in the value ( $x_{2}$ in the notation of \$2) for $Y$ that would make them indifferent. Similarly, $x_{3}$ and $x_{4}$ were elicited. The elicited values were written on a separate sheet so that the subjects could see their previous choices.

Most subjects asked for the probabilities of carrying either disease. They were informed that the people who display their symptoms are usually older than the subjects themselves. And of those older people, about $66 \%$ carry the unfavorable disease 1 (with shorter life durations). The subjects were informed that for young people like themselves the probability of carrying disease 1 could be expected to be smaller than 0.66 . They were told that this was all the information that was available about probabilities. To avoid misunderstanding, let us repeat that we do not think that unknown probabilities will in general give better utility elicitations. To the contrary, we think that better results would have been ob- 
tained in our experiment if probabilities had been provided. Our motivation for running the experiment without giving probabilities is to demonstrate the applicability of the TO method to that context. In other words, we tested the method in as difficult a context as possible. We expect that for most subjects the TO method works best with given probabilities, but for some it will not.

Stimuli for the CE Elicitations. Next a bisection CE elicitation was carried out on the same subjects using the questions described in Figure 2. Each subject's value for $x_{4}$ that was elicited earlier by the TO method was substituted in the figure. Obviously, that value differed across subjects. As before, two simple preliminary choice questions were asked to familiarize the subjects with the setting (in one choice question the value 7 was substituted for $Y$, in the other choice question the value $x_{4}-1$ was substituted). After these preliminary questions, the subjects were asked to fill in the value for $Y$ that would make them indifferent between the two operations. We denote this value here by $\mathrm{CE}\left(\frac{1}{2}\right)$. In the next question the value $x_{4}$ of the first question was replaced by the value $\operatorname{CE}\left(\frac{1}{2}\right)$ just elicited, and the value $\operatorname{CE}\left(\frac{1}{4}\right)$ indifferent to $\left(6, \frac{1}{2} ; \operatorname{CE}\left(\frac{1}{2}\right)\right)$ was then elicited. The final question elicited the value $\mathrm{CE}\left(\frac{3}{4}\right)$ that is indifferent to $\left(x_{4}, \frac{1}{2} ; \mathrm{CE}\left(\frac{1}{2}\right)\right)$.

Stimuli for the PE Elicitations. Twenty subjects were also available for PE questions (see Figure 3). The values $x_{1}$ and $x_{4}$ obtained from the TO elicitation were substituted, and the probability $p=\operatorname{PE}\left(x_{1}\right)$ was elicited to give indifference. Next $x_{2}$ and $x_{3}$ were substituted for $x_{1}$ to elicit the probabilities $\operatorname{PE}\left(x_{2}\right)$ and $\operatorname{PE}\left(x_{3}\right)$ such that $x_{j} \sim\left(x_{4}, \operatorname{PE}\left(x_{j}\right) ; x_{0}\right)$ also for $j=2,3$.

Results and Discussion of Expected Value. One of the 54 subjects in the life-duration experiment stated that he could not answer the questions. Five subjects violated monotonicity, suggesting that they had not properly understood the questions. Finally, three subjects had not responded to all questions. All of these nine subjects were discarded. The high percentage (16.7\%) of discarded subjects is a consequence of the various tests of monotonicity and the complicated chained nature of the experiment.
Four more subjects were discarded because there was no value $x_{4}$ that would make them indifferent in the last question. This is a consequence of the boundedness of the life-duration variable and the time spans we chose. We could have avoided the nonexistence of $x_{4}$ by using smaller time spans. It is well-known, however, that utility is almost linear over small intervals. Hence, for curvature of utility to be nonnegligible, the replies should cover a significant part of the subjects' (mostly aged around 25) envisioned life duration, which explains why no smaller time spans were used. Ultimately, then, the responses of 41 subjects were retained for further analysis in the life-duration experiment.

For all three sets of measurements (TO, CE, PE), no significant differences were observed between the psychology students, the economics students, and the medical residents. This may, of course, be due to the small numbers of subjects. As a result, their responses have been pooled. The average responses are given in Table 1.

The averages of the TO and $\mathrm{CE}$ results are somewhat biased downwards because the four highest replies (for which no value for $x_{4}$ exists) were discarded. The "trimmed" TO means, which are obtained by also discarding the four subjects with lowest $x_{4}$ values, may therefore provide better representations. The resulting means are then $x_{1}=11.59, x_{2}=17.49, x_{3}=24.00, x_{4}$ $=33.05$. The related CE means for the remaining subjects are then also somewhat higher, i.e., $\operatorname{CE}\left(\frac{1}{4}\right)=10.32$, $\mathrm{CE}\left(\frac{1}{2}\right)=15.68, \mathrm{CE}\left(\frac{3}{4}\right)=22.24$; the averages for the PE elicitations are all the same as before.

Under linear utility as in expected value, the TO results should satisfy $x_{1}-x_{0}=x_{2}-x_{1}=x_{3}-x_{2}=x_{4}$ $-x_{3}$. This was found for nine subjects. The average values revealed diminishing marginal utility ("risk aversion"), i.e., the intervals $x_{1}-x_{0}, x_{2}-x_{1}, x_{3}-x_{2}, x_{4}-x_{3}$ are ascending. It may be useful to note here that the values elicited by the TO (and CE) questions are inverses of utility. Diminishing marginal utility is also confirmed in a sign test: there were 17 subjects with $x_{2}-x_{1}>x_{1}$ $-x_{0}$ and 9 subjects with the opposite strict inequality $(\alpha=0.1)$; there were 18 subjects with $x_{3}-x_{2}>x_{2}-x_{1}$ and 10 with the opposite strict inequality $(\alpha=0.1)$; for 20 subjects, $x_{4}-x_{3}>x_{3}-x_{2}$ and for 2 the reversed strict inequality held ( $\alpha=0.001)$. 
Figure 2

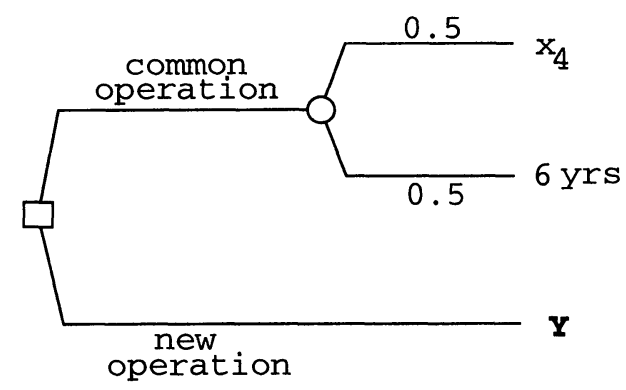

Question 1. Decide for each $6<\mathbf{Y}<\mathrm{x}_{4}$ whether you prefer the new or the common operation.

Question 2. For large values of $\mathbf{Y}$ you probably preferred the new operation, for small values the common one. Thus, for some value of $\mathbf{Y}$, your preference switched from one operation to the other. Fill in this switching value on the answer sheet.

Also in the CE questions, nine subjects maximized expected value, six of whom had done so in the TO questions too. The $\mathrm{CE}$ averages again exhibit diminishing marginal utility. Diminishing marginal utility was also revealed by the PE averages, that were taken over the 20 subjects who were available for the PE questions. If the subjects use expected value to answer the PE questions, then $\operatorname{PE}\left(x_{j}\right)=\left(x_{j}-x_{0}\right) /\left(x_{4}-x_{0}\right)$ for all $j$. Remarkably, all 20 subjects had larger PE values for $j=1$, 2 , and 18 subjects for $j=3$.

Discussion of Expected Utility. By comparing the TO results to the CE and the PE results, EU can be tested. For a person who perfectly satisfies EU in the TO and $\mathrm{CE}$ questions, the equalities $x_{j}=\mathrm{CE}(j / 4)$ will hold as both values have utility $j / 4$, for all $j$. The first three entries in Table 2 show that these EU predictions are all rejected in favor of the alternative hypotheses indicated on top. Thus, the utilities derived from the $\mathrm{CE}$ questions are more concave ("risk averse") than those derived from the TO questions.

For 19 of the 41 subjects, all three inequalities $x_{1}$ $>\mathrm{CE}\left(\frac{1}{4}\right), x_{2}>\mathrm{CE}\left(\frac{1}{2}\right), x_{3}>\mathrm{CE}\left(\frac{3}{4}\right)$ hold, and for six subjects the three reversed inequalities hold ( $p<0.01$, binomial).
If a person perfectly satisfies $\mathrm{EU}$ in the TO and PE questions, then the equalities $u\left(x_{j}\right)=j / 4$ imply that $\operatorname{PE}\left(x_{j}\right)=j / 4$ for all $j$. The last three entries in Table 2 show that these EU predictions are all rejected strongly in favor of the alternative hypotheses indicated on top. This confirms previous findings that utilities elicited through the PE method suggest very high risk aversion, i.e., (under EU) very concave utility (Officer and Halter 1968; Hershey and Schoemaker 1985; Slovic et al. 1990; Delquié 1993; Stiggelbout et al. 1994). Here 14 of the 20 subjects satisfied all three inequalities $\operatorname{PE}\left(x_{1}\right)>0.25$, $\operatorname{PE}\left(x_{2}\right)>0.50$, and $\operatorname{PE}\left(x_{3}\right)>0.75$, whereas no single subject satisfied all three inequalities $0.25 \geq \operatorname{PE}\left(x_{1}\right), 0.50$ $\geq \operatorname{PE}\left(x_{2}\right)$, and $0.75 \geq \operatorname{PE}\left(x_{3}\right)(p<0.001)$.

\subsection{The Monetary Experiment}

Subjects. The second experiment elicited utility of money from 42 subjects, consisting of 14 researchers in finance at the University of Mannheim and 28 undergraduate students in economics at the University of Limburg in Maastricht.

Method. The subjects were told that they could choose between two types of investments in a foreign

\section{Figure 3}

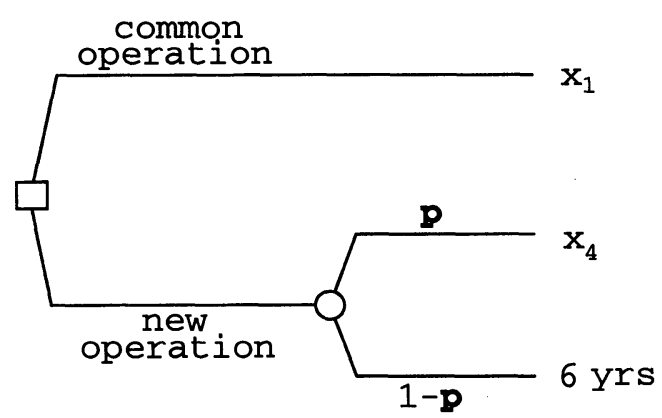

Question 1. Decide for each $\mathbf{p}=0,0.01,0.02, \ldots, 0.99,1$ whether you prefer the new or the common operation.

Question 2. For large probabilities p you probably preferred the new operation, for small probabilities the common one. Thus, for some probability p, your preference switched from one operation to the other. Fill in this switching probability on the answer sheet. 
Table 1

Average Results From the Life-duration Experiment with Standard Deviations between Brackets

\begin{tabular}{cccccc}
\hline$j$ & 0 & 1 & 2 & 3 & 4 \\
\hline \multirow{2}{*}{ TO's $x_{i}$} & 6 & 11.3 & 17.0 & 23.1 & 31.5 \\
& $(0)$ & $(2.49)$ & $(4.42)$ & $(6.68)$ & $(11.7)$ \\
CE $(j / 4)$ & - & 10.1 & 15.2 & 21.4 & - \\
& & $(2.06)$ & $(3.96)$ & $(6.36)$ & \\
$\operatorname{PE}\left(x_{i}\right)$ & - & 0.535 & 0.698 & 0.875 & - \\
& & $(0.18)$ & $(0.17)$ & $(0.13)$ & \\
\hline
\end{tabular}

country, the return of which depended on which of two possible candidates would win the upcoming elections. The unit of payment is DM 1000 for the researchers (1 DM is worth approximately $\$ 0.63$ ) and Dfl 1000 for the students ( $1 \mathrm{Dfl}$ is worth approximately $\$ 0.57$ ). As in the life-duration experiment, high outcomes were chosen to guarantee that curvature of utility would not be negligible.

Stimuli for the TO Elicitations. The format of the TO questions is displayed in Figure 4. As before, no probabilities were provided. The subjects were told that the party of candidate 1 had been more successful in the past but was running a poor election campaign this time around. As a result, it was not clear who would win the elections. In the first experimental question, the value 100 was substituted for $x_{j}$ in Figure 4 (so $x_{0}=100$ ), and subjects were asked to fill in the amount for $Y$ that would make them indifferent. This amount is $x_{1}$. In the second experimental question, the amount $x_{1}$ was substituted for $x_{j}$ in the figure, and the amount $x_{2}=Y$ that generates indifference was elicited. Similarly, $x_{3}$ and $x_{4}$ were elicited.
Figure 4

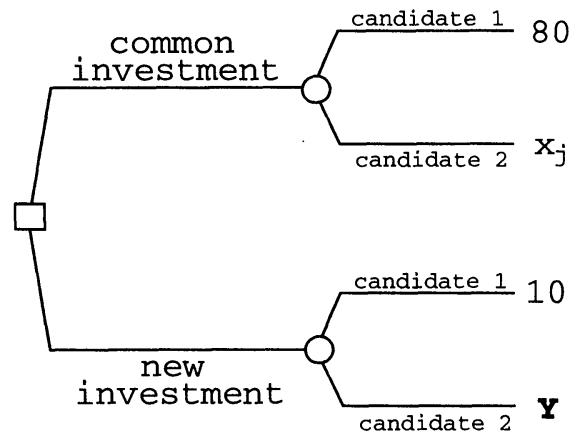

Question 1. Decide for each $100<\mathbf{Y}<1,000$ whether you prefer the new or the common investment.

Question 2. For large values of $\mathbf{Y}$ you probably preferred the new investment, for small values the common one.

Thus, for some value of $\mathbf{Y}$, your preference switched from one investment to the other. Fill in this switching value on the answer sheet.

Stimuli for the CE Elicitations. As in the life-duration experiment, $\mathrm{CE}$ questions were asked to elicit $\operatorname{CE}\left(\frac{1}{4}\right), \operatorname{CE}\left(\frac{1}{2}\right)$, and $\operatorname{CE}\left(\frac{3}{4}\right)$ for the lottery outcomes $x_{0}, x_{4}$ (Figure 5 illustrates $\mathrm{CE}\left(\frac{1}{2}\right)$ ).

Stimuli for the PE Elicitations. The PE method was tested in the monetary experiment for all students and all researchers but one. The probabilities $\operatorname{PE}\left(x_{j}\right)$ were elicited that yield indifferences $x_{j} \sim\left(x_{4}, \operatorname{PE}\left(x_{j}\right) ; x_{0}\right), j$ $=1,2,3$. See Figure 6 for $j=1$.

Results and Discussion of Normalization. One researcher and four students were discarded from the analysis because they violated monotonicity. An additional student was discarded because she provided

Table 2 Tests of EU in the Life-duration Experiment

\begin{tabular}{lccccc}
\hline$x_{1}>\mathrm{CE}\left(\frac{1}{4}\right)$ & $x_{2}>\mathrm{CE}\left(\frac{1}{2}\right)$ & $x_{3}>\mathrm{CE}\left(\frac{3}{4}\right)$ & $\mathrm{PE}\left(x_{1}\right)>\frac{1}{4}$ & $\mathrm{PE}\left(x_{2}\right)>\frac{1}{2}$ & $\mathrm{PE}\left(x_{3}\right)>\frac{3}{4}$ \\
\hline$t(40)=2.86^{\star \star}$ & $t(40)=2.10^{\star}$ & $t(40)=2.03^{\star}$ & $t(19)=7.17^{\star \star \star}$ & $t(19)=5.27^{\star \star \star}$ & $t(19)=4.19^{\star \star \star}$ \\
\hline $\begin{array}{c}\text { Paired samples } t \text {-test, one-tailed; }{ }^{\star}: p<0.05 ;{ }^{\star \star}: p<0.01 ;{ }^{\star \star \star}: p<0.001 . \text { Upper row indicates alternative } \\
\text { hypotheses. }\end{array}$
\end{tabular}


Figure 5

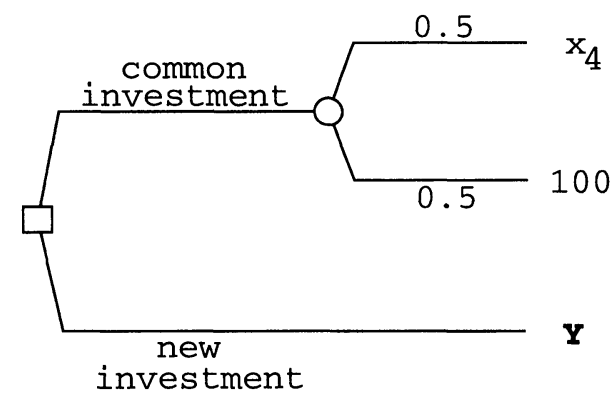

Question 1. Decide for each $100<\mathbf{Y}<\mathrm{x}_{4}$ whether you prefer the new or the common investment.

Question 2. For large values of $\mathbf{Y}$ you probably preferred the new investment, for small values the common one. Thus, for some value of $\mathbf{Y}$, your preference switched from one investment to the other. Fill in this switching value on the answer sheet.

incomplete answers. Thus the responses of 13 researchers and 23 students were retained for further analysis.

Before discussing the results of this experiment, we should note that these cannot be compared with the results of the life-duration experiment, as the two experiments concern different domains of outcomes. A comparison becomes possible if a suitable normalization is performed, and this is the topic of the following subsection. Normalization also is necessary to compare the various results of the monetary experiment with each other. One reason is that the students faced a unit of payment (Dfl 1000) that was somewhat different than that of the researchers (DM 1000). Another reason is that students' valuation of money can be expected to be considerably different than the researchers' valuation. The marginal utility of money above 100 , relative to the marginal utility between 10 and 80 , is typically smaller for students than for researchers. Therefore students require higher returns on their new investment if candidate 2 were to win to make up for the loss that would be incurred if candidate 1 were to win.

A statistical analysis confirms for each TO and CE question that the answers of the students are higher than those of the researchers. For the researchers, the average values of $x_{1}$ through $x_{4}$ are 215.38, 345.38, 503.08, and 709.23. For the students these four values are significantly higher, i.e., 306.96, 540.26, 822.43, and 1133.48 (independent samples one-tailed t-test, $p<0.05$ for all four values). Similarly, the averages of the $C E$ elicitations are all significantly higher for the students than for the researchers. These differences confirm that the responses of the students and the researchers should not be pooled.

We normalized the TO values $x_{j}$ into $n x_{j}=\left(x_{j}-x_{0}\right) /$ $\left(x_{4}-x_{0}\right)$ for $j=1,2,3$. Similarly, the CE values $\mathrm{CE}(j / 4)$ were normalized to

$$
n \mathrm{CE}(j / 4)=\left(\mathrm{CE}(j / 4)-x_{0}\right) /\left(x_{4}-x_{0}\right) .
$$

(In the notation $n x_{j}$ and $n \mathrm{CE}, n$ abbreviates "normalized" and does not designate a natural number.) After these normalizations, there were no significant differences between students' and researchers' responses. Also, there were no significant differences between their PE values. Hence the $n \mathrm{TO}, n \mathrm{CE}$, and PE values of the two groups have been pooled. Averages are given in Table 3. Let us mention here that the analysis of the lifeduration experiment could also have been performed

\section{Figure 6}

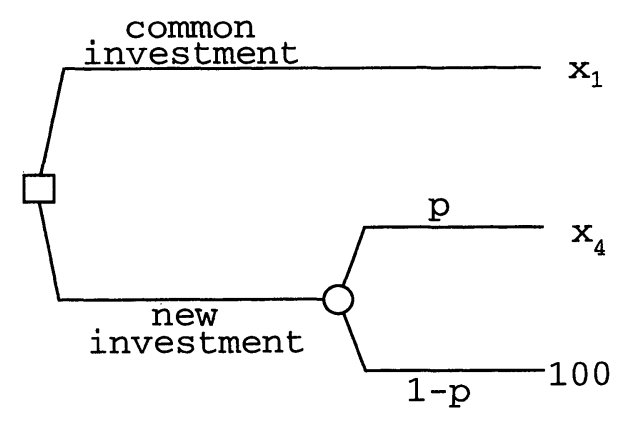

Question 1. Decide for each $\mathbf{p}=0,0.01,0.02, \ldots, 0.99,1$ whether you prefer the new or the common investment.

Question 2. For large probabilities $\mathbf{p}$ you probably preferred the new investment, for small probabilities the common one. Thus, for some probability $\mathbf{p}$, your preference switched from one investment to the other. Fill in this switching probability on the answer sheet. 


\begin{tabular}{cccc} 
Table 3 & $\begin{array}{l}\text { Average (Normalized) Results from the } \\
\text { Monetary Experiment with Standard } \\
\text { Deviations between Brackets }\end{array}$ \\
\hline$j$ & 1 & 2 & 3 \\
\hline TO's $n x_{j}$ & 0.238 & 0.475 & 0.730 \\
& $(0.11)$ & $(0.13)$ & $(0.11)$ \\
$n C E(j / 4)$ & 0.216 & 0.462 & 0.697 \\
& $(0.09)$ & $(0.12)$ & $(0.14)$ \\
$\mathrm{PE}\left(x_{j}\right)$ & 0.358 & 0.563 & 0.793 \\
& $(0.19)$ & $(0.19)$ & $(0.15)$ \\
\hline
\end{tabular}

with normalized values. That would not have affected any of our qualitative conclusions.

Discussion of Expected Value. The normalized values of the TO and CE elicitation provide an index of risk aversion within the interval $\left[x_{0}, x_{4}\right]$. Higher risk aversion implies lower normalized values. In the TO questions, three researchers and four students exhibited linear utility functions, consistent with expected value maximization. This corresponds with normalized values $n x_{1}=\frac{1}{4}, n x_{2}=\frac{1}{2}$, and $n x_{3}=\frac{3}{4}$. The average observed values are smaller, agreeing with risk aversion and diminishing marginal utility. In the $\mathrm{CE}$ questions, two researchers and nine students maximized expected value (corresponding again with normalized values $n \operatorname{CE}\left(\frac{1}{4}\right)$ $\left.=\frac{1}{4}, n \mathrm{CE}\left(\frac{2}{4}\right)=\frac{2}{4}, n \mathrm{CE}\left(\frac{3}{4}\right)=\frac{3}{4}\right)$. The two researchers, and four of these nine students, had also done so in the TO elicitation. Again, the average observed values are smaller, agreeing with risk aversion. The PE averages satisfy $\operatorname{PE}\left(x_{j}\right)>\left(x_{j}-x_{0}\right) /\left(x_{4}-x_{0}\right)$ for all $j$, which again confirms risk aversion.

Discussion of Expected Utility. Under EU, the normalized $C E$ values should be identical to the normalized TO values. They, however, turn out to be somewhat lower, although the differences are not significant; see Table 4. (The averages of nonnormalized CE values are even higher than those of the nonnormalized TO values.) For 10 subjects all three inequalities $x_{1}>\operatorname{CE}\left(\frac{1}{4}\right), x_{2}$ $>\mathrm{CE}\left(\frac{1}{2}\right)$, and $x_{3}>\mathrm{CE}\left(\frac{3}{4}\right)$ were satisfied (five researchers and five students), but for nine subjects (three researchers and six students) all of the reversed inequalities were satisfied.
The average probabilities elicited in the PE elicitations are higher than the EU predictions $\frac{1}{4}, \frac{1}{2}, \frac{3}{4}$, and these differences constitute again significant deviations from expected utility.

\subsection{Comparison of the Two Experiments}

Next we compare the results of the life-duration experiment and the monetary experiment. The averages of the normalized values are given in Table 5, and are illustrated in Figure 7 below. ${ }^{4}$ The averages of the normalized TO values of the life-duration experiment are somewhat lower than those of the monetary experiment, suggesting more risk aversion for life duration, but the differences are not significant. Also, the averages of the normalized $\mathrm{CE}$ values are lower in the lifeduration experiment, again suggesting more risk aversion than in the monetary experiment; here, the differences are large enough to be significant. Finally, the averages of the PE values are significantly higher for the life-duration experiment, once again confirming higher risk aversion there.

\subsection{Discussion of Results}

Summary of Findings. Our experiments have confirmed the general finding of diminishing marginal utility both for life years and for money. The TO elicitations suggest that the outcomes chosen in our experiments, coincidentally, exhibit similar curvature of utility for life duration as for money. Whereas under EU, the $\mathrm{CE}$ and PE elicitations should yield the same utilities as the TO elicitations, the observed $\mathrm{CE}$ elicitations suggest more concavity of utility than the TO elicitations, and the PE elicitations in turn suggest more concavity than the $\mathrm{CE}$ elicitations. In the monetary experiment, the differences between the CE elicitations and the TO elicitations are not significant and thus do not imply rejection of EU. In the life-duration experiment, the CE utilities do suggest significantly more risk aversion than the TO utilities. In both experiments, the PE utilities suggest more risk aversion than the CE utilities. PE utilities also suggest more risk aversion than $\mathrm{TO}$ utilities in the monetary

\footnotetext{
${ }^{4}$ It is remarkable that the $\mathrm{CE}$ curve for money between $\mathrm{CE}\left(\frac{1}{4}\right)$ and $\mathrm{CE}\left(\frac{3}{4}\right)$ suggests increasing marginal utility. This deviation of linearity is nonsignificant $(t(35)=0.93, p=0.359$, two-tailed).
} 


\begin{tabular}{lccccc}
\hline$n x_{1}>n \mathrm{CE}\left(\frac{1}{4}\right)$ & $n x_{2}>n \mathrm{CE}\left(\frac{1}{2}\right)$ & $n x_{3}>n \mathrm{CE}\left(\frac{3}{4}\right)$ & $\mathrm{PE}\left(x_{1}\right)>\frac{1}{4}$ & $\mathrm{PE}\left(x_{2}\right)>\frac{1}{2}$ & $\mathrm{PE}\left(x_{3}\right)>\frac{3}{4}$ \\
\hline$t(35)=0.82^{n^{\mathrm{ns}}}$ & $t(35)=0.41^{\mathrm{ns}}$ & $t(35)=1.01^{\mathrm{ns}}$ & $t(34)=3.34^{\star *}$ & $t(34)=2.00^{\star}$ & $t(34)=1.69^{*}$ \\
\hline Paired samples $t$-test, one-tailed; ns: nonsignificant for $\alpha=0.05 ;{ }^{*}: p<0.05 ;{ }^{* *}: p<0.01$. Upper row indicates \\
alternative hypotheses.
\end{tabular}

experiment and much more risk aversion in the lifeduration experiment. For a person who perfectly satisfies $\mathrm{EU}$, the three utility curves for life duration would coincide, and so would the three utility curves for money in Figure 7. Hence our findings constitute violations of EU.

Factors Inducing Violations of EU. Two important reasons for deviations from $\mathrm{EU}$ have been proposed in the literature: (1) probability distortion, and (2) response mode effects. Probability distortion is most pronounced for probabilities near 0 and 1, leading to the certainty effect (Kahneman and Tversky 1979, Tversky and Kahneman 1992). ${ }^{5}$ Riskless gambles, yielding an outcome with probability 1 , occur both in the CE and the PE questions. We can thus expect significant certainty effects in our experiments. This explanation suggests that the utilities elicited by the $C E$ and PE method are too risk averse.

We now turn to the response mode effects. In the $\mathrm{CE}$ questions, subjects were asked to respond in terms of outcomes (money or life duration), in the PE questions they had to respond in terms of probabilities. The "scale compatability effect," an example of a response mode effect, suggests that subjects pay more attention to stimulus features that resemble the response scale. Therefore, they pay more attention to the probabilities in the PE elicitations and dislike more the uncertainty of the risky choice. This could explain why the PE elicitations suggest higher risk aversion. ${ }^{6}$

\footnotetext{
${ }^{5}$ The certainty effect relates to the relative overvaluation of riskless gambles in comparison to risky gambles.

${ }^{6}$ Response mode effects have been used to explain preference reversals in other contexts. For extensive studies see Slovic et al. (1990), Fischer and Hawkins (1993), and Delquié (1993).
}

Implications for the Elicitation Methods. The above explanations are in line with the findings of the experiments. Thus a possible interpretation of our findings is as follows: The TO method has elicited curvature of utility for life duration and for money. In the domains of our experiments, the two curvatures happen to be similar. The CE method is distorted by the certainty effect, which is more pronounced in the life durationexperiment than in the monetary experiment. Therefore, the $\mathrm{CE}$ elicitations in the life duration-experiment deviate significantly from the $\mathrm{TO}$ elicitations and also from the $\mathrm{CE}$ elicitations in the monetary experiment. The PE method is subject to probability distortion, as is the CE method, and therefore exhibits similar characteristics. In addition, the PE method is distorted by response mode effects so that its results deviate more from EU than the $\mathrm{CE}$ results.

Subjects are more risk averse for life years than for money. While the classical EU conclusion would be that utility for life duration is more curved than for money, our analysis concludes that curvature of utility is similar in the two experiments. The difference lies in the deviation from EU, which is more pronounced in the lifeduration experiment.

To conclude, deviations from EU seem to affect the $\mathrm{CE}$ and PE results while the TO method seems to be insensitive to such deviations. A theoretical foundation for this claim is derived in the following section.

\section{Eliciting Utility for Nonexpected Utility Theories}

This section demonstrates that the TO method can elicit utility for some non-EU models. We mainly discuss rank-dependent utility theory and cumulative prospect theory. We show that for these theories the TO method 


\begin{tabular}{|c|c|c|c|c|c|c|c|c|c|}
\hline & $n x_{1}$ & $n x_{2}$ & $n x_{3}$ & $n \mathrm{CE}\left(\frac{1}{4}\right)$ & $n C E\left(\frac{1}{2}\right)$ & $n C E\left(\frac{3}{4}\right)$ & $\operatorname{PE}\left(x_{1}\right)$ & $\operatorname{PE}\left(x_{2}\right)$ & $\operatorname{PE}\left(x_{3}\right)$ \\
\hline years & $0.227^{\mathrm{ns}}$ & $0.458^{\mathrm{ns}}$ & $0.695^{\text {ns }}$ & $0.177^{\star}$ & $0.392^{\star}$ & $0.630^{\star}$ & $0.535^{\star \star *}$ & $0.698^{\star \star}$ & $0.875^{\star}$ \\
\hline money & 0.238 & 0.475 & 0.730 & 0.216 & 0.462 & 0.679 & 0.358 & 0.562 & 0.793 \\
\hline
\end{tabular}

Independent samples $t$-test, two-tailed; ns: nonsignificant for $\alpha=0.05 ;{ }^{\star}: p<0.05 ;{ }^{\star \star}: p<0.01 ;{ }^{\star \star \star}: p<0.001$.

completely eliminates the distortions of utility measurement due to nonlinearity of probability.

As two-outcome gambles are sufficient for elicitation purposes, we limit the description of the theories to those gambles. General definitions for multi-outcome gambles are given by Wakker and Tversky (1993) and

Figure 7 Utility Curves Derived from Average (Normalized) Values under Expected Utility

\begin{tabular}{|c|c|c|c|}
\hline elicitation method & TO & CE & PE \\
\hline line & - & $\ldots . .$. & -- \\
\hline utility for life duration & $U_{f f}(\mathrm{TO})$ & $U_{l f}(\mathrm{CE})$ & $U_{i f}(\mathrm{PE})$ \\
\hline utility for money & $U_{\$}(\mathrm{TO})$ & $U_{\$}(\mathrm{CE})$ & $U_{\$}(\mathrm{PE})$ \\
\hline coordinates in plane & $\left(x_{j}, \frac{j}{4}\right)$ & $\left(\mathrm{CE}\left(\frac{j}{4}\right), \frac{j}{4}\right)$ & $\left(x_{j}, \operatorname{PE}\left(x_{j}\right)\right)$ \\
\hline symbol for life duration & $=$ & $\square$ & 图 \\
\hline
\end{tabular}

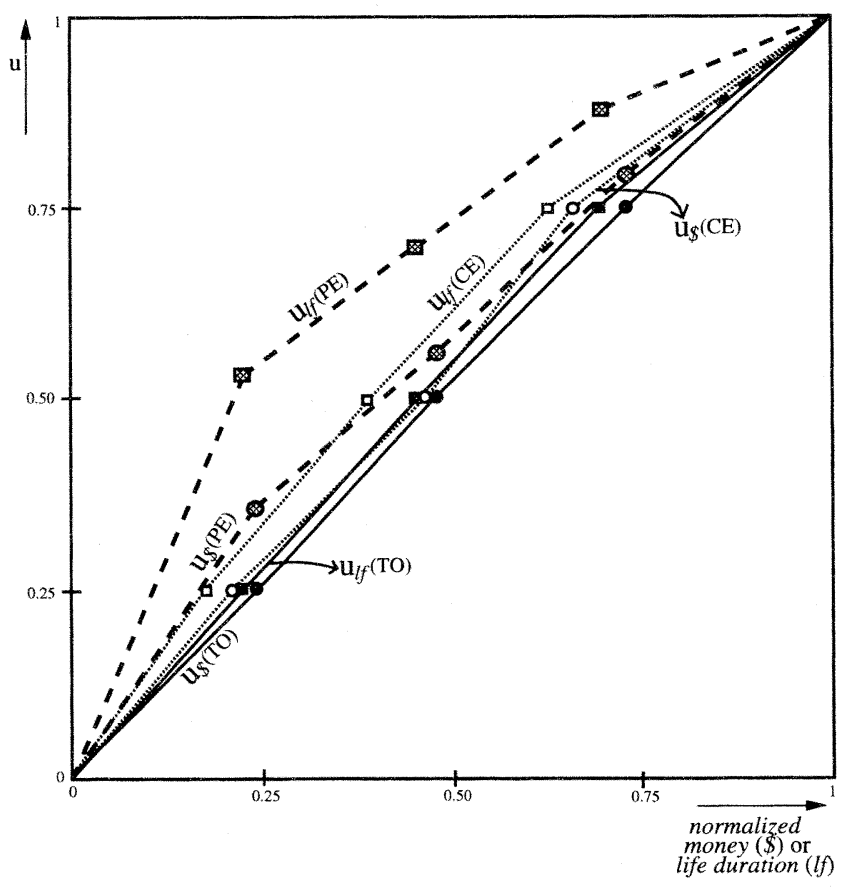

many other references. Consider our life-duration experiment, where gambles of the form $(X, A ; x)$ were used; here $A$ stood for the event "disease 1." Assume first that these gambles are valued by a "weighting" formula

$$
s \times u(X)+t \times u(x),
$$

where $s$ and $t$ are positive and depend on $A$ and (not $-A$ ). The most well-known case is, of course, expected utility where $s$ and $t$ are probabilities and sum to 1 . Another example is the approach, studied in Edwards (1962) and other papers, where $s$ and $t$ are "transformed probabilities." This approach applies to the case where objective probabilities $p$ and $1-p$ are given for $A$ and $($ not $-A)$. Then $s=f(p)$ and $t=f(1-p)$ for a probability transformation function $f$, and $s$ and $t$ need not sum to 1 .

Assume again that the indifferences $(X, A ; r) \sim(x, A$; $R)$ and $(Y, A ; r) \sim(y, A ; R)$ hold as in Equation (2). Under the weighted valuation proposed above, these indifferences still have the same implications as we derived before under the EU valuation: They now imply the equalities $s u(X)+t u(r)=s u(x)+t u(R)$ and $s u(Y)$ $+t u(r)=s u(y)+t u(R)$. Then $s(u(X)-u(x))=s(u(Y)$ - $u(y))$, and we conclude that $u(X)-u(x)=u(Y)$ $-u(y)$. This is the same conclusion as under EU. Hence the TO method can be used for utility elicitation under these more general theories.

Unfortunately, as was discovered by Fishburn (1978), weighting formulas as in Formula (3) above, where the weights need not sum to 1 , violate basic principles such as stochastic dominance. Therefore new generalizations of EU have been developed, where weights depend on the rank-ordering of outcomes in such a manner that they always sum to 1 . This idea was developed by Quiggin (1981) in the context of decision under risk, where objective probabilities are given. Schmeidler (1989) 
developed essentially the same idea in the context of decision under uncertainty, where probabilities need not be known. We refer to their theories as rankdependent utility. Tversky and Kahneman (1992), and others, used the idea of rank-dependence to improve the theoretical part of prospect theory, leading to cumulative prospect theory. Here not only rank-dependence but also "sign-dependence" is relevant. As all outcomes in our experiments are assumed of the same sign, signdependence does not affect the valuations and the derivation below holds both for rank-dependent utility and for cumulative prospect theory.

Under rank-dependence, gambles are valued as in Formula (3) above, but the weights $s$ and $t$ can depend on the ordering of $X$ and $x$. They can be different when $X \geq x$ than when $X<x$. They always sum to 1 , so that we can substitute $t=1-s$. Thus, if $X \geq x$ then the gamble $(X, A ; x)$ is valued by

$$
s \times u(X)+(1-s) \times u(x),
$$

and if $X<x$ the gamble is valued by

$$
s^{\prime} \times u(X)+\left(1-s^{\prime}\right) \times u(x),
$$

where $s \neq s^{\prime}$ is permitted. Note that, if $X=x$, either formula can be applied because both formulas yield the same result. ${ }^{7}$

In the experiments, indifferences $\left(x_{j}, A ; r\right) \sim\left(x_{j-1}, A\right.$; $R$ ) were considered $(j=1, \ldots, 4)$ where always $x_{j} \geq r$, $x_{j-1} \geq R$. In rank-dependent utility theory such gambles, which have the same ordering of outcomes, are called comonotonic. We can therefore adopt the valuation of Equation (4) with weight $s$ for all experimental gambles, and the indifferences imply

$$
s\left(u\left(x_{j+1}\right)-u\left(x_{j}\right)\right)=(1-s)(u(R)-u(r)),
$$

for all $j$. From this it follows that

$$
s\left(u\left(x_{1}\right)-u\left(x_{0}\right)\right)=\cdots=s\left(u\left(x_{4}\right)-u\left(x_{3}\right)\right),
$$

hence

$$
u\left(x_{1}\right)-u\left(x_{0}\right)=\cdots=u\left(x_{4}\right)-u\left(x_{3}\right) .
$$

\footnotetext{
${ }^{7}$ In the context of risk, where a probability $p$ for $A$ is given, the rankdependent theories assume that $s=f(p)$ and $s^{\prime}=1-f(1-p)$ for a "probability transformation" function $f$. In the context of uncertainty, $s=W(A)$ and $s^{\prime}=1-W($ not $-A)$ for a weighting function $W$.
}

If we set $u\left(x_{0}\right)=0$ and $u\left(x_{4}\right)=1$ then $u\left(x_{1}\right)=\frac{1}{4}, u\left(x_{2}\right)$ $=\frac{2}{4}, u\left(x_{3}\right)=\frac{3}{4}$. Hence the utility elicitations of the TO method in our experiments retain full validity under rank-dependent utility and cumulative prospect theory! It means that the TO curves in Figure 7 are also valid utility curves under these non-EU theories (the other curves are not).

Let us next discuss the general TO indifferences

$$
\left(x_{1}, A ; r\right) \sim\left(x_{0}, A ; R\right), \ldots,\left(x_{n}, A ; r\right) \sim\left(x_{n-1}, A ; R\right) .
$$

Under rank-dependent utility, setting $u\left(x_{0}\right)=0, u\left(x_{1}\right)$ $=1 / n$, these indifferences still imply that $u\left(x_{j}\right)=j / n$ for all $j$ as long as all gambles have the same rankordering of outcomes. That is, either $r<R<x_{0}$ or $x_{n}$ $<r<R$. Thus the TO method can be used under rankdependent utility if one takes the values $R, r$ outside of the domain of the $x_{j}$ 's where utility should be elicited, i.e., $R$ and $r$ are uniformly greater or smaller than the values $x_{j}$. Under cumulative prospect theory, an additional restriction should be imposed in view of sign dependence, that is, the $x_{j}^{\prime}$ s should all be positive or they should all be negative.

Utility elicitation for rank-dependent theories is discussed in Quiggin (1981; 1992, §10.7) and Mangelsdorff and Weber (1994). They invoke the simplifying assumption that probabilities $1 / 2$ are not transformed. This means that for an event $B$ with probability $1 / 2$, gambles $(X, B ; x)$ are evaluated by $1 / 2 \times u(X)+1 / 2$ $\times u(x)$, as they would be under EU. Then the CE method of EU can be used to elicit utility. Empirical investigations of probability transformation have found that, on average, probabilities below $1 / 3$ are transformed upward, probabilities above $1 / 3$ are transformed downward, and the probability $1 / 3$ is not transformed. Hence Tversky and Fox (1995) elicited utilities from gambles $(X, B ; x)$ where $X>x$ and the probability of $B$ is $1 / 3$ instead of $1 / 2$. It seems that, on average, the probability $1 / 3$ is more suited for utility elicitation than the more commonly used probability $1 / 2$. Still, gambles with probability $1 / 3$ do not eliminate distortions due to probability transformations at the individual level. The TO method does provide elimination of those distortions at the individual level.

Once utilities have been elicited, they can be used to elicit decision weights and thereby the probability trans- 
formation function. Thus the TO method can be used to elicit the entire Choquet expected utility model and, obviously, the entire EU model if EU is assumed.

Finally, we briefly show that the TO method can also elicit utility if utility depends on the state of nature. Assume that a gamble $(X, A ; x)$ is evaluated by the form $P(A) U_{A}(X)+P($ not $-A) U_{\text {not }-A}(x)$. This generalizes expected utility by permitting utility to be state-dependent, i.e., utility depends on the associated event and $U_{A}$ can be different from $U_{\text {not }-A}$. An example where this is reasonable is when, before a trip to Japan, event $A$ predicts that the exchange rate will increase by two cents tomorrow, and event not- $A$ predicts that it will remain unchanged. Now consider the indifferences in Equation (2). From substitution it can be derived that these indifferences still imply the equality $U_{A}(X)-U_{A}(x)$ $=U_{A}(Y)-U_{A}(y)$. The TO method thus elicits standard sequences for the state-dependent utilities, and statedependent utility functions can still be elicited up to a scale and location parameter. If the standard sequences for utility differ significantly for different events, then state-dependence has been detected.

It is impossible, under state-dependent expected utility, to compare the scale parameters for the functions $U_{A}$ and $U_{\text {not-A }}$ above and, in general, for utility functions associated with different events. Therefore no probabilities can be elicited. The nonseparability of probabilities and utility for state-dependent expected utility is central in Nau (1994).

\section{Discussion: Advantages of the Trade-off Method over Other Methods}

We already mentioned the validity problem of direct scaling. An additional problem is that the results of direct scaling seem to be more susceptible to domain and framing effects (Nord 1992, p. 560 and Fischer 1995). We shall not discuss direct scaling further.

Instead, we turn to the PE, CE, and lottery-equivalent methods. As these methods assume EU, they are susceptible to the many violations of EU that have been reported in the literature. In particular, they use probabilities in the calculations and require precise knowledge of those probabilities. This assumes that clients un- derstand and correctly perceive probabilities, which has been a central problem in utility elicitation. Consequently, these methods cannot be used for events with unknown or ambiguous probabilities. As pointed out above, the TO method can be used in such situations.

To avoid the distortions due to the certainty effect, variations of the $C E$ and $P E$ method have been proposed in which a client compares two lotteries that both involve risk, and then substitutes one of the outcomes or one of the probabilities to obtain indifference (Farquhar 1984). The most well-known variation is the lotteryequivalent method introduced by $\mathrm{McC}$ ord and de Neufville (1986). It uses indifferences $(X, p ; 0) \sim(x, q ; 0)$, for $0 \neq p \neq 1$ and $0 \neq q \neq 1$, to elicit utility. Thus it reduces the disturbing effects of probability distortion but does not entirely discard it, and still requires precise knowledge of probability.

An earlier study that also avoided the certainty effect was Davidson et al. (1957), who formalized and applied the early ideas of Ramsey (1931). They used events with probability $\frac{1}{2}$ (elicited from preference-symmetry) and used indifferences $\left(X, \frac{1}{2} ; x\right) \sim\left(Y, \frac{1}{2} ; y\right)$ to elicit equalities $u(X)-u(Y)=u(x)-u(y)$. Six outcomes were elicited that are equally spaced in utility. For each subject, only one choice was between a certain outcome and a lottery, all other questions concerned two-lottery choices. Extensive cross-checkings were done. Next, by means of the elicited utilities, subjective probabilities of events were elicited.

Note that the probability $\frac{1}{2}$ is crucial in the Ramsey method. Prospect theory suggests that the obtained utility values will still overestimate the degree of risk aversion because the probability $\frac{1}{2}$ is transformed downward by the average subject.

Like the methods described above, the TO method avoids the certainty effect by using choices between two gambles rather than choices between a gamble and a certain outcome. In addition, it does not only reduce, but completely circumvents errors due to probability distortion. A disadvantage of "two-gamble" approaches is that processing two gambles is somewhat more complicated than processing a gamble and a certain outcome. We think, however, that the TO questions appeal to a natural intuition as subjects make decisions by weighing positive and negative arguments: they are 
asked how much they need to gain in one event to match a loss in another event. As a result, we expect that the TO method will discourage the use of ad hoc decision heuristics (von Winterfeldt and Edwards 1986, $\S 10.3$; Tversky et al. 1988; Fischer 1995).

Another advantage of the TO method is that gambles with specified probabilities need not be invoked. Such gambles may feel artificial and are problematic for subjects who are not familiar with probabilities. The elicitation questions under the TO method can refer to events that are of interest to the client (" $X$ if the market goes up, $r$ if it does not"). Probabilities of such events are usually not known (contrary to the probabilities of lottery-wheel events). Dyckman and Salomon (1972) have suggested that artificial lotteries lead to distortions. They found that utility functions elicited by random devices such as colored chips in a box were rather different, usually displaying more risk-aversion than those based on simulations of actual decision situations.

It had been known before that extraneous random devices with known probabilities are not needed to axiomatize EU. The first demonstration had been given by Savage in $1954 .^{8}$ The TO method provides a similar result for utility elicitation.

The TO method is also well-suited for correcting measurement errors because cross-checkings are easily developed. For example, for any reference outcomes $r, R$ by means of which $x_{0}, \ldots, x_{n}$ are elicited, we can fix an outcome $s$ that is different from $r$ and ask the client for the outcome $S>s$ such that the gambles $\left(x_{1}, A ; s\right)$ and $\left(x_{0}, A ; S\right)$ are indifferent. Then we can use $s, S$ instead of the reference outcomes $r, R$ and verify the indifferences $\left(x_{i+1}, A ; s\right) \sim\left(x_{i}, A ; S\right)$. Under EU these indifferences should hold for all $i$.

Suppose for example that in the experiment in $\S 3$ we find $\left(x_{1}, A ; 0\right) \sim\left(x_{0}, A ; 2\right)$ for a subject, where $A$ describes the event that the patient carries disease 1 . Then

\footnotetext{
${ }^{8}$ The earlier attempt of Ramsey (1931) was incomplete, the approach of de Finetti (1937) had the drawback of assuming that utilities were known, and the approaches of von Neumann and Morgenstern (1944) and Anscombe and Aumann (1963) had the drawback of assuming given probabilities. Recent axiomatizations that avoid Savage's requirement of infinite state spaces are given by Wakker (1984, 1989, 1993) and Gul (1992).
}

we can take $s=0, S=2$, and verify the indifferences $\left(x_{2}, A ; 0\right) \sim\left(x_{1}, A ; 2\right),\left(x_{3}, A ; 0\right) \sim\left(x_{2}, A ; 2\right)$, and $\left(x_{4}, A\right.$; $0) \sim\left(x_{3}, A ; 2\right)$. If the client never deviates from $\mathrm{EU}$ then these indifferences should all hold. In practice, they can be used to independently measure the standard sequence and can help to improve the accuracy of measurement.

Note that the presence of inconsistencies in an individual's choices is a major reason for the existence of elicitation methods and decision analysis. Elicitation methods can help detect and remove inconsistencies that are due to human error. Prescriptive decision analysis asks a client to resolve the inconsistencies detected by cross-checkings, rather than accept and study them through error theories as done in descriptive approaches (Laskey and Fischer 1987, §6.3). In addition, clients find utility elicitation more acceptable when they are informed of their inconsistencies and are encouraged to modify their expressed preferences (Kimbrough and Weber 1994).

Larger utility intervals can be used for cross-checking. If in the experiments of $\S 3$ we were to find $\left(x_{2}, A ; 0\right)$ $\sim\left(x_{0}, A ; 5\right)$ for a subject, then under EU we should also find $\left(x_{3}, A ; 0\right) \sim\left(x_{1}, A ; 5\right)$ and $\left(x_{4}, A ; 0\right) \sim\left(x_{2}, A ; 5\right)$. These indifferences could then be used for a crosschecking. Note that we used zero years versus five years given not- $A$, instead of two years versus five years as in $\S 3$. One can also check for standardsequences conditional on other events or other verbal or numerical uncertainties. For instance, one can interchange the events $A$ and not- $A$, and use indifferences of the sort $\left(t, A ; x_{i+1}\right) \sim\left(T, A ; x_{i}\right)$, for $i=1, \ldots$, $n-1$, to elicit the standard sequence and for further cross-checkings. A "top down" elicitation can be used as an alternative. Then one starts with $x_{n}$, and uses indifferences $\left(x_{j}, A ; r\right) \sim\left(x_{j-1}, A ; R\right)$ to elicit $x_{n-1}$, $\ldots, x_{0}$.

The TO method is also suited for theoretical work, and can be used to reformulate an axiomatic foundation of EU described by Wakker $(1984,1989)$ and Wakker and Tversky (1993). There EU was axiomatized by a "TO consistency" axiom. From that axiomatization it easily follows that a person violates EU if and only if inconsistencies can be produced by the TO elicitation procedure. In practice, EU will be discarded if the de- 
viations from expected utility, uncovered by crosscheckings, are too large.

Another feature of the TO method is that it strongly appeals to the intuition of strength of preference. The indifferences $\left(x_{0}, A ; 5\right) \sim\left(x_{1}, A ; 2\right)$ and $\left(x_{i}, A ; 5\right) \sim\left(x_{i+1}\right.$, $A ; 2)$ in the experiments, for $i=1,2,3$, suggest that the strength of preference of $x_{i+1}$ over $x_{i}$ is as large as the strength of preference of $x_{1}$ over $x_{0}$. These indifferences are used to elicit corresponding equalities of utility differences. Still, the elicited utilities have been derived solely from "ordinal" indifferences and are not susceptible to the methodological criticisms of approaches that take strength of preference as a directly observable primitive. The elicited utilities do have full validity as vNM utilities. Thus the method may provide a bridge between risky cardinal utility and "value functions" that are to represent strengths of preferences (Dyer and Sarin 1982, Barron et al. 1984, Wakker 1984).

For simplicity, we presented the TO method for quantitative outcomes. It can also be used when outcomes are not quantified. Whatever the outcomes $R, r$ and $X$, $x, Y, y$ are in Equation (2), the indifferences elicit the equality $U(X)-U(x)=U(Y)-U(y)$. The outcomes need not be final and definite, but may involve uncertainty and risk. Some flexibility concerning the outcome space is, however, required. In axiomatic analyses, a topological "connectedness" condition (Wakker 1989) or an algebraic "solvability" condition (Wakker 1991) suffice. For the TO elicitation procedure in $\$ 2$ it is necessary that the outcomes $x_{0}, x_{1}, \ldots, x_{n}$, satisfying the required indifferences are available. A similar richness condition is required for the $\mathrm{CE}$ method. The PE method does not require richness of outcomes, but instead requires richness of the conceivable probabilities, which seems to be more convenient.

The TO method provides alternative means of eliciting qualitative properties of utility. For instance, diminishing marginal utility ("risk aversion") can be inferred if and only if all elicited standard sequences $x_{0}, \ldots, x_{n}$ have increasing step sizes $x_{j+1}-x_{j}$. The utility is a linear/exponential function if and only if standard sequences are invariant under addition of a constant ("constant absolute risk aversion," Keeney and Raiffa 1976). Utility is a $\log$ / power function if and only if elicited standard sequences are invariant under multipli- cation by positive constants ("constant relative risk aversion," Keeney and Raiffa 1976). These results also hold true for rank-dependent utility and cumulative prospect theory. Characterizations of parametric families of utility for nonexpected utility models have been studied by Miyamoto (1988), Wakker and Tversky (1993), Miyamoto and Wakker (1996), Dyckerhoff (1994), and Fishburn (1995).

In a mathematical sense, the TO method can be considered to be a special case of multi-attribute utility theory where dimensions now refer to different resolutions of uncertainty. A topic that has been discussed extensively in multiattribute utility is the issue of distortions of attribute weights (Weber et al. 1988, Tversky et al. 1988, Fischer and Hawkins 1993, von Nitzsch and Weber 1993). A recent survey has been given by Borcherding et al. (1995). In our context the attribute weights are the probabilities. These distortions of attribute weights / probabilities do not by themselves constitute a problem for the TO method. Only when the distortion effects change during the elicitation process, can they affect the results of the TO method. As an example, in the experiments we used matching judgments. It has been found that these lead to an overweighting of the dimension where the matching is to be done, and to excessive attention to the scale to be matched. However, this distortion is the same for all revealed values, and therefore does not affect the elicited utility values.

\section{Disadvantages of the Tradeoff Method}

A disadvantage of the TO method is that it is somewhat more laborious than existing methods. In each choice, clients are confronted with two gambles rather than with a gamble and a certain outcome. Therefore, subjects will initially find the TO method harder to understand. This was indeed found in the experiments. We expect, however, that after some practice subjects will find the TO method easier to deal with than the CE or PE methods. A first reason is that the new matching value that is elicited per choice question is always based on a comparison with the same "reference" outcomes under the opposite event. In the life-duration experiment, the reference outcomes were always two years 
and five years under disease 2 , in the monetary experiment they were 10 and 80 under candidate 1 . A second reason is that attention is focused on utility.

Von Winterfeldt and Edwards $(1986, \S 10.3)$ pointed out that the CE method has the disadvantage of inducing subjects to anchor on expected value calculations, which can distort their responses. The TO method may have a similar disadvantage because an easy response strategy for subjects is to simply let the difference between $x_{j}$ and $x_{j-1}$ be the same for all $j$. In our experiments, 16 of the 81 subjects used the mentioned response strategy in the TO questions, while 20 subjects resorted to expected value calculations in the $C E$ questions.

The TO method is also more laborious because, to elicit $n-1$ utility values, ${ }^{9} n$ indifferences must be observed. The CE and PE method need one observation less to elicit the same number of utilities. This is so because the $\mathrm{CE}$ and PE methods assume one additional parameter given: the probability. Often a specific parametric family has already been deemed appropriate for the utility, and utility elicitation is only used to determine the parameter(s) of the parametric family. For instance, if a power family $u(x)=x^{r}$ is chosen, then only a few preference indifferences need to be observed for the assessment of the ("risk") parameter $r$ (for multiattribute utility theory, compare Kirkwood and Sarin 1980). The TO method can also be used to identify such parameters, again at the expense of additional questions but with more robustness.

Another disadvantage of the TO method is that responses are "chained": previously elicited values must be invoked to elicit new utility values. The elicitation of $x_{3}$ requires the value $x_{2}$ as an input, etc. Responses are also chained in the bisection version of the CE method. They are neither in the basic CE method nor in the PE method. The problem with chaining is that the elicitation procedure is more complicated and that errors propagate. The error in the elicitation of, say, the ninth value can be expected to be three times larger than the error in the elicitation of the first value if errors are in-

\footnotetext{
${ }^{9}$ That is, $u\left(x_{2}\right), \ldots, u\left(x_{n}\right)$, after arbitrarily setting $u\left(x_{0}\right)=0, u\left(x_{1}\right)$ $=1 / n$.
}

dependent and identically distributed. Cross-checkings can, however, easily be developed for the TO method to reduce those errors.

Finally, only one boundary value can be fixed for the TO method, such as the minimal starting level $x_{0}$ in the description of $\S 1$, or a maximal starting level in the "top down" method described in $§ 5$. The decision analyst cannot entirely control the location of the other boundary value that will be elicited.

\section{Conclusion}

This paper has discussed the tradeoff method for eliciting von Neumann-Morgenstern utilities. The method is robust against distortions of probabilities and can be applied when probabilities are ambiguous or unknown. Probabilities can even be presented verbally. Also, the method can be adapted to rank-dependent nonexpected utility. Experiments suggest that the tradeoff method is less affected by deviations from EU than the CE and PE methods. The primary application of the tradeoff method lies in prescriptive decision analysis, where rational and truly representative utilities are to be constructed under guidance of a decision analyst, and where the method appeals to a rational decision process: the weighing of arguments. ${ }^{10}$

${ }^{10}$ The authors appreciate the helpful comments made by Hein Fennema, Bob Nau, Rakesh Sarin, Anne Stiggelbout, Lia Verhoef, and Bob Winkler.

\section{References}

Allais, M., "Le Comportement de l'Homme Rationnel devant le Risque: Critique des Postulats et Axiomes de l'Ecole Américaine," Econometrica, 21 (1953), 503-546.

$\rightarrow$ Anscombe, F. J. and R. J. Aumann, "A Definition of Subjective Probability," Ann. Math. Statistics, 34 (1963), 199-205.

Barron, H. F., D. von Winterfeldt, and G. W. Fischer, "Theoretical and Empirical Relationships between Risky and Riskless Utility Functions," Acta Psychologica, 56 (1984), 233-244.

Birnbaum, M. H. and S. E. Sutton, "Scale Convergence and Utility Measurement," Organizational Behavior and Human Decision Processes, 52 (1992), 183-215.

Borcherding, K., S. Schmeer, and M. Weber, "Biases in Multiattribute Weight Elicitation," in J.-P. Caverni, M. Bar-Hillel, F. H. Barron, and H. Jungermann (Eds.), Contributions to Decision Making-I, Elsevier, Amsterdam, 1995, 3-28. 
Davidson, D., P. Suppes, and S. Siegel, "Decision Making: An Exper- $\rightarrow$ Johnson, R. M., "Trade-off Analysis of Consumer Values," J. Marketing imental Approach," Stanford University Press, Stanford, CA, 1957. Chapter 2 reprinted in E. Edwards and A. Tversky (1967, Eds.), Decision Making, Penguin, Harmondsworth, 170-207.

de Finetti, B., "La Prévision: Ses Lois Logiques, ses Sources Subjectives," Annales de l'Institut Henri Poincaré 7, 1-68. Translated into English by H. E. Kyburg, "Foresight: Its Logical Laws, its Subjective Sources," in H. E. Kyburg and H. E. Smokler (1964, Eds.), Studies in Subjective Probability, Wiley, New York, 53-118; 2nd edition 1980, Krieger, New York.

Delquié, P., "Inconsistent Trade-Offs between Attributes: New Evidence in Preference Assessment Biases," Management Sci., 39 (1993), 1382-1395.

Dyckerhoff, R., "Decomposition of Multivariate Utility Functions in Non-Additive Expected Utility Theory," J. Multi-Criteria Decision Analysis, 3 (1994), 41-58.

Dyckman, T. R. and R. Salomon, "Empirical Utility Functions and Random Devices: An Experiment," Decision Sci., 3 (1972), 113.

$\rightarrow$ Dyer, J. S., P. C. Fishburn, R. E. Steuer, J. Wallenius, and S. Zionts, "Multiple Criteria Decision Making, Multiattribute Utility Theory: The Next Ten Years," Management Sci., 38 (1992), 645-654.

$-\rightarrow$ and R. K. Sarin, "Relative Risk Aversion," Management Sci., 28 (1982), 875-886.

Edwards, W., "Subjective Probabilities Inferred from Decisions," Psychological Review, 69 (1962), 109-135.

$\rightarrow$ Farquhar, P., “Utility Assessment Methods," Management Sci., 30 (1984), 1283-1300.

Fischer, G. W., "Range Sensitivity of Attribute Weights in Multiattribute Utility Assessment," Organizational Behavior and Human Decision Processes, 62 (1995), 252-266.

— and S. A. Hawkins, "Strategy Compatibility, Scale Compatibility, and the Prominence Effect," J. Experimental Psychology: Human Perception \& Performance, 19 (1993), 580-597.

$\rightarrow$ Fishburn, P. C., "Methods of Estimating Additive Utilities," Management Sci., 13 (1967), 435-453.

- , "On Handa's New Theory of Cardinal Utility' and the Maximization of Expected Return," J. Political Economy, 86 (1978), 321324.

—, "Nonlinear Preference and Utility Theory," Johns Hopkins University Press, Baltimore, MD, 1988.

_ , "Utility of Wealth in Nonlinear Utility Theory," AT\&T Bell Laboratories, Murray Hill, NJ, 1994.

- "Utility of Wealth in Nonlinear Utility Theory," in C. Dowling, F. Roberts, and P. Theuns (Eds.), Recent Progress in Mathematical Psychology, Erlbaum, Hillsdale, NJ, 1995.

Gul, F., "Savage's Theorem with a Finite Number of States," J. Economic Theory, 57 (1992), 99-110.

$\rightarrow$ Hershey, J. C. and P. H. J. Schoemaker, "Probability versus Certainty Equivalence Methods in Utility Measurement," Management Sci., 31 (1985), 1213-1231.

$\rightarrow$ Hogarth, R. M. and H. J. Einhorn, "Venture Theory: A Model of Decision Weights," Management Sci., 36 (1990), 780-803. Research, 11 (1974), 121-127.

Kahneman, D. and A. Tversky, "Prospect Theory: An Analysis of Decision under Risk," Econometrica, 47 (1979), 263-291.

Karni, E. and D. Schmeidler, "Utility Theory with Uncertainty," in W. Hildenbrand and H. Sonnenschein (Eds.), Handbook of Mathematical Economics, Volume 4, North-Holland, Amsterdam, 1990.

Keeney, R. L. and H. Raiffa, Decisions with Multiple Objectives, Wiley, New York, 1976.

Kimbrough, S. O. and M. Weber, "An Empirical Comparison of Utility Assessment Programs," European J. Oper. Res., 75 (1994), 617-633.

$\rightarrow$ Kirkwood, C. W. and R. K. Sarin, "Preference Conditions for Multiattribute Value Functions," Oper. Res., 28 (1980), 225-232.

Kischka, P. and C. Puppe, "Decisions under Risk and Uncertainty: A Survey of Recent Developments," Zeitschrift für Oper. Res., 36 (1992), 125-147.

Krantz, D. H., R. D. Luce, P. Suppes, and A. Tversky, Foundations of Measurement-Volume I, Additive and Polynomial Representations, Academic Press, New York, 1971.

$\rightarrow$ Laskey, K. B. and G. W. Fischer, "Estimating Utility Functions in the Presence of Response Error," Management Sci., 33 (1987), 965-980.

Loomes, G. and L. McKenzie, "The Use of QALYs in Health Care Decision Making," Social Sci. and Medicine, 28 (1989), 299-308.

$\rightarrow$ Machina, M. J., " 'Expected Utility' Analysis without the Independence Axiom," Econometrica, 50 (1982), 277-323.

$\rightarrow-$, "Decision-Making in the Presence of Risk," Science, 236 (1987), 537-543.

$\rightarrow$ McCord, M. and R. de Neufville, " 'Lottery Equivalents': Reduction of the Certainty Effect Problem in Utility Assessment," Management Sci., 32 (1986), 56-60.

Mangelsdorff, L. and M. Weber, "Testing Choquet Expected Utility," J. Economic Behavior and Organization, 25 (1994), 437-457.

Miyamoto, J. M., "Generic Utility Theory: Measurement Foundations and Applications in Multiattribute Utility Theory," J. Math. Psychology, 32 (1988), 357-404.

$\rightarrow$ and P. P. Wakker, "Multiattribute Utility Theory without Expected Utility Foundations," Oper. Res., 44 (1996), 313-326.

Nau, R., "Coherent Decision Analysis with Inseparable Probabilities and Utilities," J. Risk and Uncertainty, forthcoming.

Nitzsch, R. von and M. Weber, "The Effect of Attribute Ranges on Weights in Multiattribute Utility Measurements," Management Sci., 39 (1993), 937-943.

Nord, E., "Methods for Quality Adjustment of Life Years," Social Sci. and Medicine, 34 (1992), 559-569.

$\rightarrow$ Officer, R. R. and A. N. Halter, "Utility Analysis in a Practical Setting," American J. Agricultural Economics, 50 (1968), 257-277.

Quiggin, J., "Risk Perception and Risk Aversion among Australian Farmers," Australian J. Agricultural Economics, 25 (1981), 160169.

_ , "Generalized Expected Utility Theory - The Rank-Dependent Model," Kluwer Academic Press, Dordrecht, 1992.

Ramsey, F. P., "Truth and Probability," in The Foundations of Mathematics and other Logical Essays, 156-198, Routledge and Kegan Paul, 
London, 1931, Reprinted in H. E. Kyburg and H. E. Smokler (1964, Eds.), Studies in Subjective Probability, Wiley, New York, 61-92.

Savage, L. J., "The Foundations of Statistics," Wiley, New York, 1954. (Second edition, Dover, New York, 1972.)

Slovic, P., D. Griffin, and A. Tversky, "Compatibility Effects in Judgment and Choice," in R. M. Hogarth (Ed.), Insights in Decision Making, A Tribute to Hillel J. Einhorn, The University of Chicago Press, Chicago, IL, 1990, 5-27.

Schmeidler, D., "Subjective Probability and Expected Utility without Additivity," Econometrica, 57 (1989), 571-587.

Stiggelbout, A. M., G. M. Kiebert, J. Kievit, J. W. H. Leer, G. Stoter, J. C. J. M. de Haes, “Utility Assessment In Cancer Patients: Adjustment of Time Tradeoff Scores for the Utility of Life Years and Comparison with Standard Gamble Scores," Medical Decision Making, 14 (1994), 82-90.

Torrance, G. W., "Social Preferences for Health States: An Empirical Evaluation of Three Measurement Techniques," Socio-Economic Planning and Sci., 10 (1976), 129-136.

Tversky, A. and D. Kahneman, "Advances in Prospect Theory: Cumulative Representation of Uncertainty," J. Risk and Uncertainty, 5 (1992), 297-323.

- and C. R. Fox, "Weighing Risk and Uncertainty," Psychological Review, 102 (1995), 269-283.

— S. Sattath, and P. Slovic, "Contingent Weighting in Judgment and Choice," Psychological Review, 95 (1988), 371-384.

von Neumann, J. and O. Morgenstern, "Theory of Games and Economic Behavior," Princeton University Press, Princeton, NJ, 1944. von Winterfeldt, D. and W. Edwards, "Decision Analysis and Behavioral Research." Cambridge University Press, Cambridge, 1986.

Wakker, P. P., "Cardinal Coordinate Independence for Expected Utility," J. Math. Psychology, 28 (1984), 110-117.

- "Additive Representations of Preferences, A New Foundation of Decision Analysis," Kluwer Academic Publishers, Dordrecht, 1989.

- "Under Stochastic Dominance Choquet-Expected Utility and Anticipated Utility are Identical," Theory and Decision, 29 (1990), 119-132.

_ , "Additive Representations of Preferences, A New Foundation of Decision Analysis; The Algebraic Approach," in J. P. Doignon and J. C. Falmagne (Eds.), Mathematical Psychology: Current Developments, Springer, Berlin, 1991, 71-87.

$\rightarrow-$, ' 'Unbounded Utility for Savage's 'Foundations of Statistics', and Other Models," Math. Oper. Res., 18 (1993), 446-485.

_- "Separating Marginal Utility and Probabilistic Risk Aversion," Theory and Decision, 36 (1994), 1-44.

- and A. Tversky, "An Axiomatization of Cumulative Prospect Theory," J. Risk and Uncertainty, 7 (1993), 147-176.

Weber, E. U., "From Subjective Probabilities to Decision Weights: The Effect of Asymmetric Loss Functions on the Evaluation of Uncertain Outcomes and Events," Psychological Bulletin, 115 (1994), 228-242.

$\rightarrow$ Weber, M., F. Eisenführ, and D. von Winterfeldt, "The Effects of Splitting Attributes on Weights in Multiattribute Utility Measurement," Management Sci., 34 (1988), 431-445.

Accepted by Gregory W. Fischer; received October 22, 1993. This paper has been with the authors 8 months for 2 revisions. 